\title{
003 PP COLLABORATING WITH YOUNG PEOPLE TO SET THE AGENDA FOR SECONDARY ANALYSIS OF THE BRIGHTLIGHT DATASET
}

RM Taylor, * A Martins, S Lea, JS Whelan, LA Fern. Cancer Division, University College London Hospitals NHS Foundation Trust, UK

10.1136/bmjopen-2017-016492.21

BRIGHTLIGHT is a national evaluation of teenage and young adult cancer services in England. Data are collected five times over three years from 830 young people using a bespoke survey. Additional data are collected from carers, clinical notes and National Health Service (NHS) registries; consequently, BRIGHTLIGHT is the most comprehensive data set from young people with cancer in the world. In addition to the primary research question such a wealth of information lends itself to further exploration.

Objective: Identify what young people think is important to investigate for secondary data analysis.

Methods: A workshop was held with eight members of the BRIGHTLIGHT user group. To facilitate young people's understanding of 'hypothesis generation', the board game Twister was adapted so each movement represented a different survey question/domain. The game was played according to the rules with the modification that after each pair of movements, participants discussed possible linkage between two domains represented by the two twister movements.

Results: Thirty-six links were discussed and refined into 21 areas for analysis. These were sent to the user group to individually vote for their top 3 :

- Is the impact of cancer affected by how much support you get from people in similar situations?

- Am I less likely to be involved in decision making if I am younger?

- How I feel about my body after cancer affects my ability to form new relationships.

Conclusions: Young people can be involved in hypothesis generation given appropriate tools and identify linkages between data not necessarily viewed as important by healthcare professionals. 